PROCEEDINGS OF THE

AMERICAN MATHEMATICAL SOCIETY

Volume 126, Number 12, December 1998, Pages 3527-3537

S $0002-9939(98) 04375-5$

\title{
ON A CONJECTURE OF F. MÓRICZ
}

\author{
G. BROWN AND K. Y. WANG
}

(Communicated by J. Marshall Ash)

Abstract. F. Móricz has investigated the integrability of double lacunary sine series. His result, valid for special lacunary sequences, does not extend in the form originally conjectured, but we establish a suitably modified result.

\section{INTRODUCTION}

Let $a_{i j}, i, j \in \mathbb{N}$, be real numbers satisfying the condition

$$
\sigma=\left\{\sum_{i=1}^{\infty} \sum_{j=1}^{\infty} a_{i j}^{2}\right\}^{\frac{1}{2}}<\infty .
$$

Suppose $q>1$ and $m_{i}, n_{j}$ are positive numbers satisfying

$$
\frac{m_{i+1}}{m_{i}} \geq q, \quad \frac{n_{j+1}}{n_{j}} \geq q, \quad m_{1}=n_{1}=1, i, j \in \mathbb{N} .
$$

Define

$$
\begin{gathered}
f(x, y)=\sum_{i=1}^{\infty} \sum_{j=1}^{\infty} a_{i j} \sin m_{i} x \sin n_{j} y \\
g_{j}(x)=\sum_{i=1}^{\infty} a_{i j} \sin m_{i} x, \quad h_{j}(y)=\sum_{j=1}^{\infty} a_{i j} \sin n_{j} y .
\end{gathered}
$$

In the general case these limits are to be understood in the sense of $L^{2}$-convergence and, as Lemma 1 shows, there is no inherent ambiguity in the definition.

F. Móricz [3] considered the special case when $m_{i}=n_{i}=2^{i-1}, i \in \mathbb{N}$. In this case he proved that the condition

$$
\sum_{i=1}^{\infty} \sum_{j=1}^{\infty}\left(\sum_{k=i}^{\infty} \sum_{l=j}^{\infty} a_{k l}^{2}\right)^{\frac{1}{2}}<\infty
$$

is equivalent to

$$
\frac{f(x, y)}{x y} \in L(0,1)^{2}, \quad \frac{g_{i}(x)}{x} \in L(0,1), \quad \frac{h_{i}(y)}{y} \in L(0,1), \quad i \in \mathbb{N} .
$$

Received by the editors June 22, 1993 and, in revised form, February 12, 1997.

1991 Mathematics Subject Classification. Primary 42B05.

Key words and phrases. Double sine series, lacunarity, integrability.

This work was supported by a grant from the Australian Research Council.

(C)1998 American Mathematical Society 
He proposed that in the general case when $m_{i}, n_{j}$ are positive integers satisfying condition (2) and the integrability condition, then (4) is satisfied if and only if

$$
\sum_{i=1}^{\infty} \sum_{j=1}^{\infty} \log \frac{m_{i+1}}{m_{i}} \log \frac{n_{j+1}}{n_{j}}\left(\sum_{k=i}^{\infty} \sum_{l=j}^{\infty} a_{k l}^{2}\right)^{\frac{1}{2}}<\infty .
$$

Our result is the following

Theorem. Let $a_{i j}, m_{i}, n_{j}$ satisfy (1) and (2). Let $f, g_{j}, h_{i}$ be as above. Define

$$
\begin{gathered}
S=\sum_{i=1}^{\infty} \sum_{j=1}^{\infty}\left|a_{i j}\right|, \quad T=\sum_{i=1}^{\infty} \sum_{j=1}^{\infty} \log \frac{m_{i+1}}{m_{i}}\left(\sum_{k=i+1}^{\infty} a_{k j}^{2}\right)^{\frac{1}{2}}, \\
U=\sum_{i=1}^{\infty} \sum_{j=1}^{\infty} \log \frac{n_{j+1}}{n_{j}}\left(\sum_{l=j+1}^{\infty} a_{i l}^{2}\right)^{\frac{1}{2}}, \\
V=\sum_{i=1}^{\infty} \sum_{j=1}^{\infty} \log \frac{m_{i+1}}{m_{i}} \log \frac{n_{j+1}}{n_{j}}\left(\sum_{k=i+1}^{\infty} \sum_{l=j+1}^{\infty} a_{k l}^{2}\right)^{\frac{1}{2}} .
\end{gathered}
$$

Then the condition (4) is equivalent to the condition

$$
S+T+U+V<\infty
$$

We point out that in our theorem, $m_{i}, m_{j}$ need not be integers. If $m_{i}=n_{i}=$ $2^{i-1}$, then (5) is equivalent to (6). But in general, as the following example shows, (5) is stronger than (6) and they are not equivalent.

First we note that the one-dimensional case is subsumed by the two-dimensional case. If we set $a_{i j}=b_{i}$ for $j=1$ and $a_{i j}=0$ for $j>1$, then (5) reads

$$
\sum_{i=1}^{\infty} \log \frac{m_{i+1}}{m_{i}}\left(\sum_{j=i}^{\infty} b_{j}^{2}\right)^{\frac{1}{2}}<\infty
$$

and (6) reads

$$
\sum_{i=1}^{\infty}\left|b_{i}\right|+\sum_{i=1}^{\infty} \log \frac{m_{i+1}}{m_{i}}\left(\sum_{j=i+1}^{\infty} b_{j}^{2}\right)^{\frac{1}{2}}<\infty
$$

Let $b_{i}=\left\{e^{-2^{i+1}}-e^{-2^{i+2}}\right\}^{1 / 2}$ and $m_{i}=\prod_{l=1}^{i} e^{e^{e^{l-1}}}$. Then we see that $\left(6^{\prime}\right)$ holds but $\left(5^{\prime}\right)$ does not.

We present the proof of the Theorem in two parts:

Theorem 1. Let $d=1+\frac{4}{(q-1)^{2}}+\frac{2}{(q-1)} \sqrt{\frac{4}{(q-1)^{2}}+8}$. Then

$$
\int_{0}^{d}\left|g_{j}(x)\right| \frac{d x}{x} \leq c_{q}\left\{\sum_{i=1}^{\infty}\left|a_{i j}\right|+\sum_{i=1}^{\infty} \log \frac{m_{i+1}}{m_{i}}\left(\sum_{k=i+1}^{\infty} a_{k j}^{2}\right)^{\frac{1}{2}}\right\},
$$




$$
\begin{gathered}
\int_{0}^{d}\left|h_{i}(y)\right| \frac{d y}{y} \leq c_{q}\left\{\sum_{j=1}^{\infty}\left|a_{i j}\right|+\sum_{j=1}^{\infty} \log \frac{n_{j+1}}{n_{j}}\left(\sum_{l=j+1}^{\infty} a_{i l}^{2}\right)^{\frac{1}{2}}\right\} \\
\int_{0}^{d} \int_{0}^{d}|f(x, y)| \frac{d x d y}{x y} \leq c_{q}(S+T+U+V) .
\end{gathered}
$$

This theorem demonstrates that (6) implies (4).

Theorem 2. (4) implies (6).

We point out that the number $d=d(q)$ in Theorem 1 is just the positive root of the equation: $\frac{1}{4}(d-1)^{2}-\frac{2}{(q-1)^{2}}(d+1)-\frac{4}{(q-1)^{2}}=0$. This particular definition of $d$ will be retained throughout the paper.

\section{Proof of Theorem 1}

Lemma 1. Let $a, b$ be arbitrary real numbers and $Q=(a, a+d) \times(b, b+d)$. Then $0.001 \sigma \leq\left\{\frac{1}{d^{2}} \int_{Q} f^{2}(x, y) d x d y\right\}^{\frac{1}{2}} \leq \sigma$.

Proof. Let $I_{i j k l}=\int_{Q} \sin m_{i} x \sin m_{k} x \sin n_{j} y \sin n_{l} y d x d y$. We have $\int_{Q} f^{2}(x, y) d x d y$ $=\sum a_{i j} a_{k l} I_{i j k l}$, where the sum $\sum$ is taken over $\mathbb{N}^{4}$. If $i \neq k$ and $j \neq l$, then

$$
\left|I_{i j k l}\right| \leq\left(\frac{1}{\left|m_{i}-m_{k}\right|}+\frac{1}{m_{i}+m_{k}}\right)\left(\frac{1}{\left|n_{j}-n_{l}\right|}+\frac{1}{n_{j}+n_{l}}\right) .
$$

Let $S_{1}$ denote the subset of $\mathbb{N}^{4}$ defined by $S_{1}=\left\{(i, j, k, l) \in \mathbb{N}^{4}: i \neq k, j \neq l\right\}$ and let $\sum_{1}$ denote the sum taken over $S_{1}$. By Schwarz's inequality $\left|\sum_{1} s_{i j} a_{k l} I_{i j k l}\right| \leq$ $\left\{\sum_{1}\left|a_{i j} a_{k l}\right|^{2}\right\}^{1 / 2}\left\{\sum_{1} I_{i j k l}^{2}\right\}^{1 / 2}$. Applying condition (2) we find $\sum_{1} I_{i j k l}^{2} \leq$ $16\left(\frac{1}{1-q^{-2}}\right)^{2}\left(\frac{1}{q^{2}-1}\right)^{2}$ and hence

$$
\left|\sum_{1} a_{i j} a_{k l} I_{i j k l}\right| \leq\left\{\frac{4}{(q-1)^{2}}-a_{q}\right\} \sigma^{2},
$$

where $a_{q}=\frac{4}{(q+1)(q-1)^{2}}$.

If $i=k$ and $j \neq l$, then $\left|I_{i j k l}\right| \leq \frac{d+1}{2}\left(\frac{1}{\left|n_{j}-n_{l}\right|}+\frac{1}{n_{j}+n_{l}}\right)$. Applying condition (2) again we find $\sum_{2}\left|a_{i j} a_{k l} I_{i j k l}\right| \leq\left\{\frac{2(d+1)}{(q-1)^{2}}-b_{q}\right\} \sigma^{2}$, where $\sum_{2}$ denotes the sum over the set $\{i \neq k, j=l\} \cup\{i=k, j \neq l\}$ and $b_{q}=\frac{2(d+1)}{(q-1)^{2}(q+1)}$.

Finally, if $(i, j)=(k, l)$, then by condition (2) we have

$$
\begin{gathered}
\frac{1}{4}\left\{d-\max \left(|\sin d|, \frac{1}{q}\right)\right\}^{2} \leq I_{i j k l} \leq \frac{1}{4}(d+1)^{2}, \\
\frac{1}{4}(d-1)^{2} \sigma^{2} \leq \sum_{i=1}^{\infty} \sum_{j=1}^{\infty} \sigma_{i j}^{2} I_{i j i j} \leq \frac{1}{4}(d+1)^{2} \sigma^{2} .
\end{gathered}
$$

Combining the estimates for $\sum_{1}, \sum_{2}$ and (10), noticing $\frac{1}{4}(d-1)^{2}-\frac{2(d+1)}{(q-1)^{2}}-\frac{4}{(q-1)^{2}}=$ 0 we get $\sqrt{a_{q}+b_{q}} \sigma \leq\left\{\int_{Q} f^{2}(x, y) d x d y\right\}^{\frac{1}{2}} \leq d \sigma$. If $1<q \leq 31$, then $\sqrt{a_{q}+b_{q}}>$ $\frac{d}{850}$ and hence $\left\{\frac{1}{|Q|} \int_{Q} f^{2}(x, y) d x d y\right\}^{\frac{1}{2}} \geq \frac{\sigma}{850}$. If $q \geq 31$, then $d<1.2$ and hence $I_{i j i j} \geq \frac{1}{4}(d-\sin 1.2)^{2}$. So we modify (10) to get

$$
\int_{Q} f^{2}(x, y) d x d y \geq\left\{a_{q}+b_{q}+0.034^{2}\right\} \sigma^{2}
$$


and hence for $q>31$ we have $\left\{\frac{1}{|Q|} \int_{Q} f^{2}(x, y) d x d y\right\}^{\frac{1}{2}}>0.028 \sigma$. The combination of these estimates completes the proof.

The following lemma is a direct corollary of Lemma 1.

Lemma 2. Let $a_{j}$ be real numbers satisfying $A=\left(\sum_{j=1}^{\infty} a_{j}^{2}\right)^{1 / 2}<\infty$ and let $m_{j}$ be numbers satisfying the condition:

$$
m_{1}=1, \quad \frac{m_{i+1}}{m_{i}} \geq q>1 .
$$

Define $\psi(x)=\sum_{j=1}^{\infty} a_{j} \sin m_{j} x$. Then for any $a \in \mathbb{R}$

$$
\frac{1}{200} A \leq\left\{\frac{1}{d} \int_{a}^{a+d} \psi^{2}(x) d x\right\}^{\frac{1}{2}} \leq 2 A .
$$

Proof of Theorem 1. We first prove (7). For fixed $j \in \mathbb{N}$, we omit the subscript $j$, and write $g=g_{j}, a_{i}=a_{i j}$ for simplicity. Then we have

$$
\int_{0}^{d} x^{-1}|g(x)| d x \leq \sum_{i=1}^{\infty} \int_{\frac{d}{m_{i+1}}}^{\frac{d}{m_{i}}} x^{-1}\left\{\left|S_{i}(x)\right|+\left|T_{i}(x)\right|\right\} d x
$$

where $S_{i}(x)=\sum_{k=1}^{i} a_{k} \sin m_{k} x, T_{i}(x)=\sum_{k=i+1}^{\infty} a_{k} \sin m_{k} x$. Since $\left|\sin m_{k} x\right| \leq$ $m_{k}|x|$ we see $\int_{\frac{d}{m_{i+1}}}^{\frac{d}{m_{i}}}\left|S_{i}(x)\right| \frac{d x}{x} \leq d \sum_{k=1}^{i}\left|a_{k}\right| \frac{1}{q^{i-k}}$, and

$$
\sum_{i=1}^{\infty} \int_{d / m_{i+1}}^{d / m_{i}} x^{-1}\left|S_{i}(x)\right| d x \leq c_{q} \sum_{i=1}^{\infty}\left|a_{i j}\right| .
$$

Simultaneously, writing $c_{k}=a_{i+k, j}, u_{k}=\frac{m_{i+k}}{m_{i+1}}$ we get

$$
\int_{\frac{d}{m_{i+1}}}^{\frac{d}{m_{i}}}\left|T_{i}(x)\right| \frac{d x}{x}=\int_{d}^{\frac{m_{i+1}}{m_{i}} d}\left|\sum_{k=1}^{\infty} c_{k} \sin u_{k} x\right| \frac{d x}{x} .
$$

Let $m=\left[\frac{m_{i+1}}{m_{i}}\right]$ and $\psi(x)=\sum_{k=1}^{\infty} c_{k} \sin u_{k} x$. Then

$$
\int_{d}^{\frac{m_{i+1}}{m_{i}} d}|\psi(x)| \frac{d x}{x} \leq \sum_{\mu=1}^{m} \int_{\mu d}^{(\mu+1) d}|\psi(x)| \frac{d x}{x} .
$$

By Lemma 2 we have $\int_{\mu d}^{(\mu+1) d}|\psi(x)| \frac{d x}{x} \leq \frac{2}{\mu}\left(\sum_{k=i+1}^{\infty} a_{k j}^{2}\right)^{1 / 2}$ and hence

$$
\begin{gathered}
\int_{\frac{d}{m_{i+1}}}^{\frac{d}{m_{i}}}\left|T_{i}(x)\right| \frac{d x}{x} \leq c_{q} \log \frac{m_{i+1}}{m_{i}}\left\{\sum_{k=i+1}^{\infty} a_{k j}^{2}\right\}^{1 / 2}, \\
\sum_{i=1}^{\infty} \int_{d / m_{i+1}}^{d / m_{i}} x^{-1}\left|T_{i}(x)\right| d x \leq c_{q} \sum_{i=1}^{\infty} \log \frac{m_{i+1}}{m_{i}}\left(\sum_{k=i+1}^{\infty} a_{k j}^{2}\right)^{1 / 2} .
\end{gathered}
$$

Combining (12), (13) and (15) we get (7). By symmetry (8) follows. 
Now we write $I_{i j}=\int_{\frac{d}{m_{i+1}}}^{\frac{d}{m_{i}}} \int_{\frac{d}{n_{j+1}}}^{\frac{d}{n_{j}}}|f(x, y)| \frac{d x d y}{x y}$ and define

$$
\begin{gathered}
\phi_{k l}(x, y)=a_{k l} \sin m_{k} x \sin n_{l} y, \\
f_{1}(x, y)=\sum_{k=1}^{i} \sum_{l=1}^{j} \phi_{k l}(x, y), \quad f_{2}(x, y)=\sum_{k=i+1}^{\infty} \sum_{l=1}^{j} \phi_{k l}(x, y), \\
f_{3}(x, y)=\sum_{k=1}^{i} \sum_{l=j+1}^{\infty} \phi_{k l}(x, y), \quad f_{4}(x, y)=\sum_{k=i+1}^{\infty} \sum_{l=j+1}^{\infty} \phi_{k l}(x, y) .
\end{gathered}
$$

Then define $I_{i j}^{(\nu)}=\int_{d / m_{i+1}}^{d / m_{i}} \int_{d / n_{j+1}}^{d / n_{j}}\left|f_{\nu}(x, y)\right| \frac{d x d y}{x y}, \nu=1,2,3,4$. We see $I_{i j}^{(1)} \leq$ $d^{2} \sum_{k=1}^{i} \sum_{l=1}^{j}\left|a_{k l}\right| \frac{1}{q^{i-k}} \cdot \frac{1}{q^{j-l}}$ and hence

$$
\sum_{i=1}^{\infty} \sum_{j=1}^{\infty} I_{i j}^{(1)} \leq c_{q} \sum_{i=1}^{\infty} \sum_{j=1}^{\infty}\left|a_{i j}\right|=c_{q} S .
$$

Secondly, $I_{i j}^{(2)} \leq \sum_{l=1}^{j} \frac{d}{q^{j-l}} \int_{\frac{d}{m_{i+1}}}^{\frac{d}{m_{i}}}\left|\sum_{k=i+1}^{\infty} a_{k l} \sin m_{k} x\right| \frac{d x}{x}$. So by (14)

$$
\int_{\frac{d}{m_{i+1}}}^{\frac{d}{m_{i}}}\left|\sum_{k=i+1}^{\infty} a_{k l} \sin m_{k} x\right| \frac{d x}{x} \leq c_{q} \log \frac{m_{i+1}}{m_{i}}\left(\sum_{k=i+1}^{\infty} a_{k l}^{2}\right)^{\frac{1}{2}} .
$$

Therefore

$$
\sum_{i=1}^{\infty} \sum_{j=1}^{\infty} I_{i j}^{(2)} \leq c_{q} T
$$

Symmetrically we have

$$
\sum_{i=1}^{\infty} \sum_{j=1}^{\infty} I_{i j}^{(3)} \leq c_{q} U
$$

Finally, to estimate $I_{i j}^{(4)}$ we change the integrating variables by writing $x=$ $s / m_{i+1}, y=t / n_{j+1}$. Then we get

$$
I_{i j}^{(4)}=\int_{d}^{\left(m_{i+1} / m_{i}\right) d} \int_{d}^{\left(n_{j+1} / n_{j}\right) d}\left|\sum_{k=1}^{\infty} \sum_{l=1}^{\infty} b_{k l} \sin u_{k} s \sin v_{l} t\right| \frac{d s d t}{s t}
$$

where $b_{k l}=a_{i+k, j+l}, u_{k}=\frac{m_{i+k}}{m_{i+1}}, v_{l}=\frac{n_{j+l}}{n_{j+1}}$. Let $m=\left[\frac{m_{i+1}}{m_{i}}\right]$ and $n=\left[\frac{n_{j+1}}{n_{j}}\right]$. Define

$$
\theta_{\mu \nu}=\int_{\mu d}^{(1+\mu) d} \int_{\nu d}^{(1+\nu) d}\left|\sum_{k=1}^{\infty} \sum_{l=1}^{\infty} b_{k l} \sin u_{k} s \sin v_{l} t\right| \frac{d s d t}{s t} .
$$

Applying Lemma 1 we get $\theta_{\mu \nu} \leq \frac{1}{\mu \nu}\left(\sum_{k=1}^{\infty} \sum_{l=1}^{\infty} b_{k l}^{2}\right)^{1 / 2}$, and hence

$$
\sum_{i=1}^{\infty} \sum_{j=1}^{\infty} I_{i j}^{(4)} \leq c_{q} V .
$$

A combination of (16)-(19) yields (9). The proof is complete. 


\section{Proof of Theorem 2}

To prove Theorem 2 we need more integral estimates.

Lemma 3. Let $m_{i}, c_{i j}, i, j \in \mathbb{N}$, be real numbers satisfying condition (11) and $B=$ $\left(\sum_{i=1}^{\infty} c_{i i}\right)^{2}+\sum_{i=1}^{\infty} \sum_{j=1}^{\infty} c_{i j}^{2}<\infty$. Define

$$
h(x)=\sum_{i=1}^{\infty} \sum_{j=1}^{\infty} c_{i j} \cos \left(m_{i}-m_{j}\right) x
$$

Then, for any $a \in \mathbb{R}, \int_{a}^{a+d} h^{2}(x) d x \leq c_{q} B$, where, and throughout this paper, $c_{q}$ denotes a constant depending only on $q$.

Proof. Define

$$
h_{1}(x)=\sum_{i=2}^{\infty} \sum_{j=1}^{i-1} c_{i j} \cos \left(m_{i}-m_{j}\right) x, \quad h_{2}(x)=\sum_{j=2}^{\infty} \sum_{i=1}^{j-1} c_{i j} \cos \left(m_{i}-m_{j}\right) x .
$$

We see $h^{2}(x) \leq 3\left\{h_{1}^{2}(x)+h_{2}^{2}(x)+\left(\sum_{i=1}^{\infty} c_{i i}\right)^{2}\right\}$. We have $h_{1}^{2}(x)=\frac{1}{2}\left\{g_{1}(x)+g_{2}(x)\right\}$ where

$$
\begin{aligned}
& g_{1}(x)=\sum_{i>j} \sum_{k>l} c_{i j} c_{k l} \cos \left(m_{i}-m_{j}-m_{k}+m_{l}\right) x, \\
& g_{2}(x)=\sum_{i>j} \sum_{k>l} c_{i j} c_{k l} \cos \left(m_{i}-m_{j}+m_{k}-m_{l}\right) x .
\end{aligned}
$$

By condition (11) for $i>j, k>l$, we have $m_{i}-m_{j}+m_{k}-m_{l} \geq 2(q-1)$. $\sqrt{m_{i-1} \cdot m_{k-1}}$. Hence by Schwarz's inequality we get

$$
\int_{d}^{a+d} g_{2}(x) d x \leq c_{q} \sum_{i=1}^{\infty} \sum_{j=1}^{\infty} c_{i j}^{2}
$$

Choose $n \in \mathbb{N}$ such that $1-\frac{1}{q}-\frac{1}{q^{n}}=\delta>0$. Then define subsets $J_{\mu}$ of $(i, j, k, l) \in \mathbb{N}^{4}$ by

$$
\begin{gathered}
J_{1}=\{i>j, i \geq k+n, k>l\}, \quad J_{2}=\{i>j, i \leq k-n, k>l\}, \\
J_{3}=\{k+n>i>k>l, i \geq j+n\}, \\
J_{4}=\{k+n>i>k>l, i>j>i-n\}, \\
J_{5}=\{i+n>k>i>j, k>l\}, \quad J_{6}=\{i=k>j, k>l\}
\end{gathered}
$$

and define $g_{1 \nu}(x)=\sum_{\nu} c_{i j} c_{k l} \cos \left(m_{i}-m_{j}-m_{k}+m_{l}\right) x, \nu=1, \ldots, 6$, where the sum $\sum_{\nu}$ denotes $\sum_{(i, j, k, l) \in J_{\nu}}$. Then we see $g_{1}=\sum_{\nu=1}^{6} g_{1 \nu}$. First we have $\int_{a}^{a+d} g_{1 l}(x) d x$ $\leq \sum_{1}\left|c_{i j} c_{k l}\right|\left(1-\frac{1}{q}-\frac{1}{q^{n}}\right)^{-1} \frac{2}{m_{i}}$. So by Schwarz's inequality

$$
\int_{a}^{a+d} g_{11}(x) d x \leq c_{q} \sum_{i=1}^{\infty} \sum_{j=1}^{\infty} c_{i j}^{2} .
$$

Symmetrically

$$
\int_{a}^{a+d} g_{12}(x) d x \leq c_{q} \sum_{i=1}^{\infty} \sum_{j=1}^{\infty} c_{i j}^{2}
$$


If $(i, j, k, l) \in J_{3}$, then $m_{i}-m_{j}-m_{k}+m_{l} \geq \delta m_{i}$. Hence we have

$$
\int_{a}^{a+d} g_{13}(x) d x \leq \frac{2}{\delta} \sum_{k=2}^{\infty} \sum_{l=1}^{k-1}\left|c_{k l}\right| \sum_{i=\max (n+1, k+1)}^{\infty} \sum_{j=1}^{i-n} \frac{\left|c_{i j}\right|}{m_{i}} .
$$

The using Schwarz's inequality we get

$$
\int_{a}^{a+d} g_{13}(x) d x \leq c_{q} B
$$

For $(i, j, k, l) \in J_{4}$ let $\varepsilon_{i j k l}=\left|\int_{a}^{a+d} \cos \left(m_{i}-m_{j}-m_{k}+m_{l}\right) x d x\right|$. Then

$$
\int_{a}^{a+d} g_{14}(x) d x \leq \sum_{k=2}^{\infty} \sum_{l=1}^{k-1} \sum_{i=k+1}^{k+n} \sum_{j=\max (1, i-n)}^{i-1}\left|c_{i j} c_{k l}\right| \varepsilon_{i j k l} .
$$

If we define $\varepsilon_{i j k l}=c_{i j}=0$ when $i \leq 0$ or $j \leq 0$, then we get

$$
\int_{a}^{a+d} g_{14}(x) d x \leq \sum_{s=1}^{n} \sum_{t=0}^{n-1} \sum_{k=2}^{\infty}\left|c_{k+s, k+s-n+t}\right| \sum_{l=1}^{k-1}\left|c_{k l}\right| \eta_{l}(k, s, t),
$$

where $\eta_{l}(k, s, t)=\varepsilon_{k+s, k+s-n+t, k, l}$ will be written as $\eta_{l}$ for simplicity. Write $\sigma_{k}=$ $\sum_{l=1}^{k-1}\left|c_{k, l}\right| \eta_{l}$. Then $\sigma_{k} \leq\left\{\sum_{l=1}^{k-1} c_{k l}^{2} \sum_{l=1}^{k-1} \eta_{l}^{2}\right\}^{1 / 2}$. Define

$$
l_{0}=\min \left\{l \in\{1, \ldots, k-1\}:\left|m_{k+s}-m_{k+s-n+1}-m_{k}+m_{l}\right|<\frac{1}{2}\left(1-\frac{1}{q}\right) m_{l}\right\},
$$

$l_{0}=0$ for the case that the minimum does not exist. As a convention we let $m_{0}=0$. Then for all $l \neq l_{0},\left|m_{k+s}-m_{k+s-n+t}-m_{k}+m_{l}\right| \geq \frac{1}{2}\left(1-\frac{1}{q}\right) m_{l}$. Hence we have $n_{l} \leq c_{q} \frac{1}{m_{l}}$, for $l \neq l_{0}, 1 \leq l \leq k-1$, and $\eta_{l_{0}} \leq d$. Consequently we get $\left\{\sum_{l=1}^{k-1} \eta_{l}^{2}\right\}^{1 / 2} \leq c_{q}$, and $\sigma_{k} \leq c_{q}\left\{\sum_{l=1}^{k-1} c_{k l}^{2}\right\}^{1 / 2}$. Now we derive

$$
\int_{a}^{a+d} g_{14}(x) d x \leq c_{q} B
$$

Since $J_{5}$ is symmetrically related to $J_{3} \cup J_{4}$ we conclude

$$
\int_{a}^{a+d} g_{15}(x) d x \leq c_{q} B
$$

By a direct calculation we get

$$
\int_{a}^{a+d} g_{16}(x) d x \leq c_{q} B
$$

A combination of (21)-(26) yields

$$
\int_{a}^{a+d} g_{1}(x) d x \leq c_{q} B
$$

Then we combine (20) and (27) to get $\int_{a}^{a+d} h_{1}^{2}(x) d x \leq c_{q} B$. Symmetrically we conclude $\int_{a}^{a+d} h_{2}^{2}(x) d x \leq c_{q} B$. And at last we derive $\int_{a}^{a+d} h^{2}(x) d x \leq c_{q} B$ as required. 
Lemma 4. Under the assumptions of Lemma 3 define

$$
g(x)=\sum_{i=1}^{\infty} \sum_{j=1}^{\infty} c_{i j} \cos \left(m_{i}+m_{j}\right) x
$$

Then $\int_{a}^{a+d} g^{2}(x) d x \leq c_{q} B$.

The proof is completely similar to that of Lemma 3 . We omit it.

Lemma 5. Under the conditions of Lemma 1

$$
\left\{\int_{Q} f^{4}(x, y) d x d y\right\} \leq c_{q} \sigma .
$$

Proof. For $i \in \mathbb{N}$ and $y \in \mathbb{R}$ fixed we define $b_{i}=\sum_{j=1}^{\infty} a_{i j} \sin n_{j} y$. Then we see $f^{2}(x, y)=\frac{1}{2}\{h(x)+g(x)\}$ where $h, g$ are defined respectively as in Lemma 3 and Lemma 4 with coefficients $c_{i j}=b_{i} b_{j}$. Then by these lemmas we conclude

$$
\int_{Q} f^{4}(x, y) d x d y \leq c_{q} \sum_{i=1}^{\infty} \sum_{j=1}^{\infty} \int_{b}^{b+d}\left\{b_{i} b_{j}\right\}^{2} d y .
$$

Since $b_{i} b_{j}=\frac{1}{2} \sum_{k=1}^{\infty} \sum_{l=1}^{\infty} a_{i j} a_{j l}\left\{\cos \left(n_{k}-n_{l}\right) y-\cos \left(n_{k}+n_{l}\right) y\right\}$ we apply Lemmas 3 and 4 again to get $\int_{b}^{b+d} b_{i}^{2} b_{j}^{2} d y \leq c_{q} \sum_{k=1}^{\infty} a_{i k}^{2} \sum_{i=1}^{\infty} a_{j l}^{2}$. Substituting this into (28) we complete the proof.

The following two estimates follow from Lemma 1 and Lemma 5.

Lemma 6. Under the conditions of Lemma $1 c_{q} \int_{Q}|f(x, y)| d x d y \geq \sigma$.

Lemma 7. Under the assumptions of Lemma $2 c_{q} \int_{a}^{a+d}|\psi(x)| d x \geq A$.

The following lemma is the essence of Móricz's Lemmas 2 and 3 of [3].

Lemma 8. Let $a_{i} \geq 0, i \in \mathbb{N}$. Then for any $r \in \mathbb{N}$ and $n>r$

$$
\sum_{i=r+1}^{n} a_{i} \leq \frac{1}{\sqrt{r}} \sum_{i=1}^{n-1}\left(\sum_{j=i+1}^{n} a_{j}^{2}\right)^{1 / 2} .
$$

Proof of Theorem 2. By Lemma 1, (4) is equivalent to

$$
\frac{f(x, y)}{x y} \in L(0, \lambda d)^{2}, \quad \frac{g_{i}(x)}{x} \in L(0, \lambda d), \quad \frac{h_{i}(y)}{y} \in L(0, \lambda d), \quad i \in \mathbb{N},
$$

where $\lambda=\max \left(1, \frac{1}{q-1}\right) \geq 1$ and $d$ is the value defined in Theorem 1 . Now assume $\left(4^{\prime}\right)$ holds; we are going to prove (6).

Let $A_{i j}=\log \frac{m_{i+1}}{m_{i}}\left(\sum_{k=i+1}^{\infty} u_{k j}^{2}\right)^{\frac{1}{2}}, B_{i j}=\log \frac{n_{j+1}}{n_{j}}\left(\sum_{l=j+1}^{\infty} a_{i l}^{2}\right)^{\frac{1}{2}}$. We first prove $\sum_{k=1}^{\infty} A_{k j}<\infty, \sum_{l=1}^{\infty} B_{i l}<\infty(i, j \in \mathbb{N})$.

For $n$ big enough and $j$ fixed, let $I_{n}=\int_{\lambda d / m_{n+1}}^{\lambda d}\left|g_{j}(x)\right| \frac{d x}{x}$. Then $I_{n} \geq J_{n}-R_{n}$ where

$$
R_{n}=\sum_{i=1}^{n} \int_{\left(\lambda d / m_{i+1}\right)}^{\left(\lambda d / m_{i}\right)}\left|\sum_{k=1}^{i} a_{k j} \sin m_{k} x\right| \frac{d x}{x} \leq c_{q} \sum_{i=1}^{n}\left|a_{i j}\right|
$$


and

$$
J_{n}=\sum_{i=1}^{n} \int_{\lambda d / m_{i+1}}^{\lambda d / m_{i}}\left|\sum_{k=i+1}^{\infty} a_{k j} \sin m_{k} x\right| \frac{d x}{x} .
$$

Applying Lemma 7 we get

$$
J_{n} \geq \sum_{i=1}^{n} \sum_{\mu=1}^{m} \frac{1}{(\mu+\lambda)} c_{q}\left(\sum_{k=i+1}^{\infty} a_{k j}^{2}\right)^{\frac{1}{2}}
$$

where $m=\left[\left(\frac{m_{i+1}}{m_{i}}-1\right) \lambda\right] \in \mathbb{N}$. It is now clear why we take $\lambda d$ instead of $d$. We obtain, in fact, $J_{n} \geq c_{q} \sum_{i=1}^{n} A_{i j}$ and hence $\sum_{i=1}^{n} A_{i j} \leq c_{q}^{\prime} \sum_{i=1}^{n}\left|a_{i j}\right|+c_{q}^{\prime \prime} I_{n}$. If $\sum_{i=1}^{\infty} A_{i j}=\infty$, noticing $\int_{0}^{\lambda d}\left|g_{j}(x)\right| \frac{d x}{x}<\infty$ we derive

$$
1 \leq \varlimsup_{n \rightarrow \infty} c_{q}^{\prime}\left\{\sum_{i=1}^{n}\left|a_{i j}\right|\left(\sum_{i=1}^{n} A_{i j}\right)^{-1}\right\}
$$

But by Lemma 8 we conclude the right part of this inequality should be zero. This contradiction shows $\sum_{i=1}^{\infty} A_{i j}<\infty$. Symmetrically we know $\sum_{j=1}^{\infty} B_{i j}<\infty$.

Next we define $f_{\nu}, \nu=1,2,3,4$, as in the proof of Theorem 1 and define

$$
E_{i j}^{(\nu)}=\int_{\left(\lambda d / m_{i+1}\right)}^{\left(\lambda d / m_{i}\right)} \int_{\left(\lambda d / n_{j+1}\right)}^{\left(\lambda d / n_{j}\right)}\left|f_{\nu}(x, y)\right| \frac{d x d y}{x y}, \quad \nu=1,2,3,4, i, j \in \mathbb{N} .
$$

For big $s, t \in \mathbb{N}$ let

$$
\sigma_{s, t}^{(\nu)}=\sum_{i=1}^{s} \sum_{j=1}^{t} E_{i j}^{(\nu)}, \quad \sigma_{s, t}=\sum_{i=1}^{s} \sum_{j=1}^{t} \int_{\lambda d / m_{i+1}}^{\lambda d / m_{i}} \int_{\lambda d / n_{j+1}}^{\lambda d / n_{j}} \frac{|f(x, y)|}{x y} d x d y .
$$

We have $\sigma_{s, t} \geq \sigma_{s t}^{(4)}-\left(\sigma_{s t}^{(1)}+\sigma_{s t}^{(2)}+\sigma_{s t}^{(3)}\right)$. By an argument similar to that used in the proof of Theorem 1 we get inequalities similar to (16)-(18), viz.

$$
\begin{gathered}
\sigma_{s t}^{(1)} \leq c_{q} \sum_{i=1}^{s} \sum_{j=1}^{t}\left|a_{i j}\right|, \quad \sigma_{s t}^{(2)} \leq c_{q} \sum_{i=1}^{s} \sum_{j=1}^{t} \log \frac{m_{i+1}}{m_{i}}\left(\sum_{k=j+1}^{\infty} a_{k j}^{2}\right)^{\frac{1}{2}}, \\
\sigma_{s t}^{(3)} \leq c_{q} \sum_{i=1}^{s} \sum_{j=1}^{t} \log \frac{n_{j+1}}{n_{j}}\left(\sum_{l=j+1}^{\infty} a_{i l}^{2}\right)^{\frac{1}{2}} .
\end{gathered}
$$

We now estimate $\sigma_{s t}^{(4)}$ applying Lemma 6 . Let $m=\left[\left(\frac{m_{i+1}}{m_{i}}-1\right) \lambda\right]$ and $n=$ $\left[\left(\frac{n_{j+1}}{n_{j}}-1\right) \lambda\right]$. Then $m \in \mathbb{N}, n \in \mathbb{N}$ and

$$
\sigma_{s t}^{(4)} \geq \sum_{i=1}^{s} \sum_{j=1}^{t} \sum_{\mu=1}^{m} \sum_{\nu=1}^{n} \int_{\lambda d+(\mu-1) d}^{\lambda d+\mu d} \int_{\lambda d+(\nu-1) d}^{\lambda d+\nu d} S_{i j}(x, y) d x d y
$$

where

$$
S_{i j}(x, y)=x^{-1} y^{-1}\left|\sum_{k=i+1}^{\infty} \sum_{l=j+1}^{\infty} a_{k l} \sin \frac{m_{k}}{m_{i+1}} x \sin \frac{n_{l}}{n_{j+1}} y\right| .
$$


Hence by Lemma 6 we get

$$
\sigma_{s t}^{(4)} \geq c_{q} \sum_{i=1}^{s} \sum_{j=1}^{t} \log \frac{m_{i+1}}{m_{i}} \log \frac{n_{j+1}}{n_{j}}\left(\sum_{k=i+1}^{\infty} \sum_{l=j+1}^{\infty} a_{k l}^{2}\right)^{\frac{1}{2}} .
$$

Then applying Lemma 8 , by an argument similar to that for the proof of $\sum_{i=1}^{\infty} A_{i j}<$ $\infty$ we conclude $V<\infty$. Then noticing

$$
\begin{aligned}
T & =\sum_{i=1}^{\infty} A_{i 1}+\sum_{i=1}^{\infty} \sum_{j=2}^{\infty} A_{i j} \leq \sum_{i=1}^{\infty} A_{i 1}+c_{q} V \\
U & =\sum_{j=1}^{\infty} B_{1 j}+\sum_{j=1}^{\infty} \sum_{i=2}^{\infty} B_{i j} \leq \sum_{j=1}^{\infty} B_{1 j}+c_{q} V, \\
S & =\sum_{j=2}^{\infty}\left|a_{i j}\right|+\sum_{i=2}^{\infty}\left|a_{i 1}\right|+\left|a_{i l}\right|+\sum_{i=2}^{\infty} \sum_{j=2}^{\infty}\left|a_{i j}\right| \\
& \leq c_{q} \sum_{j=1}^{\infty} B_{1 j}+c_{q} \sum_{i=1}^{\infty} A_{i 1}+\left|a_{11}\right|+c_{q} V,
\end{aligned}
$$

we derive (6). The proof is complete.

Remarks. (a) In our argument the series by which we define functions need not be trigonometric series because the coefficients $m_{i}, n_{j}$ need not be integers. We must understand such series in the sense of $L^{2}$-convergence. For example, if conditions (1) and (2) are satisfied, then the "partial sums"

$$
S_{\mu \nu}(x, y)=\sum_{i=1}^{\mu} \sum_{j=1}^{\nu} a_{i j} \sin m_{i} x \sin n_{j} y
$$

converge in $L^{2}(Q)$ for any compact set $Q \subset \mathbb{R}^{2}$. This is a consequence of Lemma 1 . Meanwhile we can easily demonstrate that the convergence of $S_{\mu \nu}$ in $L^{2}(Q)$ does not depend on the manner in which $\mu$ and $\nu$ tend to infinity. For a discussion of different kinds of multiple limits we refer the reader to [4].

(b) Since $S_{\mu \nu}$ can be non-trigonometric sums it does not appear to be a trivial question whether the convergence of $S_{\mu \nu}$ in $L^{2}$ implies almost everywhere convergence.

(c) Our result can be extended to higher dimensional cases in a quite straightforward manner.

(d) Since this paper was submitted in June, 1993, two related papers of interest have appeared, viz. [1], [2]. We thank the referee for providing the details.

\section{REFERENCES}

[1] C.-P. Chen, Integrability of multiple trigonometric series and parseval's formula, J. Math. Anal. Appl. 186 (1994), 182-199. MR 95h:42011

[2] — Weighted integrability and $l^{1}$-convergence of multiple trigonometric series, Studia Math. 108 (1994), 177-190. MR 95b:42010

[3] F. Móricz, Integrability of double lacunary sine series, Proc. of the AMS 110 (1990), 355-364. MR 90m: 42020 
[4] L. V. Zhizhiashvili, Certain questions from the theory of simple and multiple trigonometric and orthogonal series, Uspekhi Mat. Nauk 28 (1973), 65-127.

Vice-Chancellor, University of Sydney, Sydney, New South Wales 2006, Australia

E-mail address: m.jackson@vcc.usyd.edu.au

Department of Mathematics, Beijing Normal University, 100871 Beijing, People's Republic of China

E-mail address: wangky@email.bnu.edu.cn 